\title{
Changes in Cartilage Metabolism in Arthritis Are Reflected by Altered Serum and Synovial Fluid Levels of the Cartilage Proteoglycan Aggrecan
}

\author{
Implications for Pathogenesis
}

\author{
A. Robin Poole, ${ }^{*}$ Mirela lonescu, * Angela Swan, ${ }^{\star}$ and Paul A. Dieppe ${ }^{\star}$ \\ * Joint Diseases Laboratory, Shriners Hospital for Crippled Children, Montreal, Quebec, Canada H3G 1A6; Division of Surgical \\ Research Department of Surgery, McGill University, Montreal, Quebec, Canada H3G 1A6; and ${ }^{\ddagger}$ Department of Rheumatology, \\ University of Bristol Medical School, Bristol Royal Infirmary, BS2 8HW Bristol, United Kingdom
}

\begin{abstract}
The metabolism of the cartilage proteoglycan aggrecan was studied in patients with osteoarthritis $(\mathrm{OA}, n=83)$, rheumatoid arthritis (RA, $n=127)$, and in controls $(n=117)$ using monoclonal antibody-based radioimmunoassays for glycosaminoglycans in the serum and synovial fluid (SF) to detect epitope 846 on chondroitin sulfate (probably only on recently synthesised molecules) and a keratan sulfate (KS) epitope AN9PI, present on intact and degraded molecules.

Epitope 846 levels were always elevated in SF over serum (mean 38-fold in OA and 8.6-fold in RA) being highest in $O A$ patients with the longest disease duration and greatest loss of cartilage, and lowest in RA joints with high leucocyte counts. Serum levels were more often elevated in RA $(56 \%)$ than in OA (19\%) and probably reflect increased aggrecan synthesis in diseased joints. KS levels were higher in SF than in serum in $69 \%$ of patients (up to 2.3 -fold); levels were inversely (OA) and directly (RA) related to SF leucocyte counts. Serum KS was reduced in both diseases and in RA was inversely related to both systemic and joint inflammation markers. SF 846 levels were inversely related to SF KS in both diseases.

These epitopes may provide a measure of the balance between cartilage synthesis and degradation in these diseases. (J. Clin. Invest. 1994. 94:25-33.) Key words: cartilage $\cdot$ aggrecan $\cdot$ rheumatoid arthritis $\cdot$ osteoarthritis

\section{Introduction}

Destruction and remodeling of articular cartilage are features of both rheumatoid arthritis (RA) ${ }^{1}$ and osteoarthritis (OA) (1). In $\mathrm{OA}$, where there is relatively little synovial inflammation, the cartilage matrix degradation is usually accompanied by enhanced synthesis of proteoglycans. This has been demonstrated
\end{abstract}

Address correspondence to Professor P. A. Dieppe, Department of Rheumatology, University of Bristol, Medical School, Bristol Royal Infirmary, BS2 8HW Bristol, U.K.

Received for publication 9 August 1993 and in revised form 15 February 1994.

1. Abbreviations used in this paper: CS, chondroitin sulfate; ECP, eosinophil cationic protein; KS, keratan sulfate; MPO, myeloperoxidase; OA, osteoarthritis; PMN, polymorphonuclear leucocytes; RA, rheumatoid arthritis; SF, synovial fluid.

J. Clin. Invest.

(C) The American Society for Clinical Investigation, Inc. 0021-9738/94/07/0025/09 $\$ 2.00$

Volume 94, July 1994, 25-33 in both the human disease $(1,2)$ and in experimental models $(3,4)$. In contrast, intra-articular inflammation is a major feature of RA, resulting in polymorphonuclear (PMN)-rich cellular exudates in the joints. But relatively little is known about cartilage proteoglycan changes in this disease, or what influence the inflammation and the effects PMN products may have on matrix synthesis. In RA there is excessive production of cytokines such as interleukin $1 \alpha$ and $1 \beta(5,6)$, and these may stimulate chondrocyte-mediated matrix degradation (7-9). There is both in vivo (10) and in vitro (11-14) evidence that these same cytokines can act as potent inhibitors of matrix synthesis, at concentrations at least an order of magnitude less than those required to stimulate degradation (11).

The extensive extracellular matrix of hyaline cartilage contains a dense network of composite collagen fibrils composed mainly of type II, together with type IX and XI collagens (9). Contained within this network are large proteoglycans (called aggrecan) many of which can aggregate with hyaluronic acid. In adult cartilage, these molecules contain a relatively large proportion of the glycosaminoglycan keratan sulfate (KS) in addition to chondroitin sulfate (CS) (15). Proteoglycans isolated from adult articular cartilage consist of partially degraded molecules containing the KS rich region of aggrecan $(16,17)$.

Previous studies of aggrecan from fetal, immature, and adult articular cartilage revealed that an epitope identified by a monoclonal antibody called 846 (which is chondroitin ABC lyase labile), is most concentrated in the fetus (18). This CS-related epitope disappears progressively with aging, alongside a relative increase in the amount of KS in cartilage; the 846 epitope is almost absent from mature adult cartilage $(17,18)$. However, in OA this epitope is usually present in increased content, located on the largest of the aggrecan molecules (17). It may be confined to the most intact and recently synthesized aggrecan molecules, and may therefore be a marker of aggrecan synthesis. Related native CS epitopes also appear in the articular cartilage of dogs with experimental OA (19), and in human OA cartilage (20). These CS epitopes, including the 846 epitope, have not been detected in other adult tissues (Poole, A. R., and A. Reiner, unpublished results ).

Using antibodies to core protein and KS epitopes on aggrecan, we and others have shown that degradation products can be found in synovial fluids (SF) and sera of patients with a variety of different types of arthritis (21-30). KS-containing fragments, measured by ELISA, have been shown to be present in increased amounts in the SF, and possibly in the serum, of patients with OA (22-25), although they may be unchanged or decreased in content in the sera of patients with RA (29, 30). Our previous studies using an immunoassay which detects relatively large PG fragments containing KS (31), revealed that there was an inverse correlation between the content of these molecules and the acute phase reactant orosomucoid in the sera 
of patients with RA (29). A similar observation has since been recorded by another group using a different KS assay (30). More recently an inverse correlation has been observed between serum KS (32) and TNFa levels, indicating an effect of inflammation on systemic cartilage metabolism. However, changes in the content of KS-rich degradation products in arthritis could also result from differences in the reactivity of antibodies to $\mathrm{KS}$ in large and small fragments, as a result of changes in epitope density, an effect that can be minimized by the use of monovalent Fab fragments (31).

In the present study, we have studied patients with RA and $\mathrm{OA}$, as well as a group of subjects without arthritis. We have shown that proteoglycan fragments bearing the CS epitope 846 , as well as KS, are detectable in sera and SFs. We demonstrate significant differences in the levels of these fragments in patients with OA and RA. The 846 epitope is strikingly elevated in sera of patients with RA in contrast to reduced KS levels. In SF the 846 epitope also shows marked elevations in this case over serum levels, and especially in OA. We have specifically investigated the hypothesis that the SF concentration of these aggrecan fragments will be related to the degree of local inflammation and severity of joint damage, whereas serum levels will relate to the degree of systemic disease activity.

\section{Methods}

Subjects. After obtaining local ethics committee approval for the study, serum samples were collected from 117 ambulatory subjects attending a blood donor center, none of whom had any evidence of arthritis or any other inflammatory disease. This control group comprised 63 women and 54 men (Table I). Serum and SF samples were also collected from patients with knee arthritis requiring withdrawal of SF for diagnostic or therapeutic reasons, the serum sample being collected at the same time that the joint was aspirated. Patients were excluded if they had had a steroid injection into the index knee joint within three months of the study; other therapy was not taken into account. There were 127 patients with active RA and synovitis of the knees ( 88 women, and 39 men [Table I] ) and 83 patients with OA of the knee and an effusion (61 women and $22 \mathrm{men}$ ). All patients had been referred to the Department of Rheumatology at the Bristol Royal Infirmary; RA was diagnosed by ACR criteria (33) and OA on the basis of use-related pain in the index joint, combined with radiological features of $\mathrm{OA}$, including definite joint space narrowing. The patient groups were further divided into three groups, according to disease duration; $58 \%$ of the RA patients had had the disease for more than $10 \mathrm{yr}, 39 \%$ for $2-10 \mathrm{yr}$, and only $3 \%$ (four subjects) for less than $2 \mathrm{yr}$. $60 \%$ of those with OA had suffered for more than $10 \mathrm{yr}, 36 \%$ for $2-10 \mathrm{yr}$, and $4 \%$ (three subjects) for less than 2 yr.

Clinical assessments. The joint from which SF was obtained was assessed for pain and evidence of local inflammation at the time of the aspiration. Pain was measured on a $0-1-2-3$ scale (none, mild, moderate, severe), and the size of any effusion, as well as local heat and tenderness on 0-1-2 scales (absent, present, severe). The presence or absence of ligamentous instability was also recorded.

Synovial fluid analyses. All SFs were aspirated from the suprapatella pouch of the knee joint using a 23 gauge needle. They were immediately transferred into sterile containers containing no anticoagulant or preservatives. The initial examination and separations were carried out within $2 \mathrm{~h}$ of collection. A few drops of the fluid were used to estimate the total and differential leucocyte counts, which were divided into three categories each as follows: (a) total leucocyte counts $<1 \times 10^{5} / \mathrm{ml}$; 2. $1 \times 10^{5}-1 \times 10^{6} / \mathrm{ml} ; 3$. $>1 \times 10^{6} / \mathrm{ml}$; or mainly lymphocytes and few PMN; $(b)$ approximately equal numbers of PMN and mononuclear cells; and $(c)$ predominantly PMN. The remaining fluid was centrifuged at $2,000 \mathrm{~g}$ for $10 \mathrm{~min}$ and the supernatants aliquoted in 1-ml samples for storage at $-70^{\circ} \mathrm{C}$ before estimation of proteoglycan fragments and inflammatory markers.
Table I. Details of Patient Groups Examined

\begin{tabular}{lccc}
\hline Age & $\begin{array}{c}\text { Non-arthritic } \\
\text { normals }\end{array}$ & Osteoarthritis & Rheumatoid arthritis \\
\hline $20-30$ & 2 & - & 2 \\
$30-40$ & 2 & - & 8 \\
$40-50$ & 25 & 5 & 7 \\
$50-60$ & 25 & 23 & 34 \\
$60-70$ & 34 & 21 & 44 \\
$70-80$ & 12 & 27 & 30 \\
$80-90$ & 16 & 7 & 2 \\
Total & 117 & 83 & 127 \\
& & & \\
\hline
\end{tabular}

Radiographs. A radiograph of the knee joint from which SF had been aspirated was obtained within three months of the time of aspiration. Different radiographic features, including joint space narrowing, erosions, and osteophytosis were scored on a $0-1-2$ scale (absent, present, severe).

Total joint score. These were determined as previously described (34). Scores for each affected joint are totaled.

Inflammation markers. In addition to assays of total and differential leucocyte counts in the SF, immunoassays of PMN and eosinophil degranulation were used. Both eosinophilic cationic protein (ECP) and myeloperoxidase (MPO) levels were estimated with assay kits obtained from Pharmacia Diagnostics (Uppsala, Sweden). In addition, serum orosomucoid levels were measured (29) to provide an index of systemic inflammation.

Immunoassays of glycosaminoglycan epitopes of the cartilage proteoglycan aggrecan. KS was assayed using a solution phase competition radioimmunoassay described for serum by Poole et al (29). We used a mouse monoclonal IgG $_{1}$ antibody AN9P1 which recognizes KS present in the proteoglycan aggrecan or fragments thereof $(16)$. The antibody was used, either as IgG, which preferentially recognizes larger fragments of aggrecan core protein bearing multiple $\mathrm{KS}$ chains, or monovalent Fab (prepared from IgG with papain digestion) which recognizes both large fragments and small fragments of core protein containing single KS chains or doublets (31). These assays both utilize human adult aggrecan radiolabeled with ${ }^{125} \mathrm{I}$ as the tracer and unlabeled aggrecan as the standard. The assays employ second step antibodies (anti-mouse IgG or anti-mouse IgG Fab) as described previously $(29,31)$. All assays were performed in triplicate. The percentage coefficient of variation (standard deviation/mean concentration of replicate values $\times 100$ ) for interassay variation of the $\mathrm{KS} \mathrm{Fab}^{\prime}$ assay of serum was $12.9 \pm 8.1 \%$ (mean \pm standard deviation, $n=52$ ) and intraassay variation was $6.7 \pm 3.8 \%$ (mean \pm standard deviation, $n=25$ ). Spiking experiments of sera involving addition up to $6.25 \mathrm{mg} / \mathrm{ml}$ of fetal human aggrecan revealed good recoveries of $100.3 \pm 3.9 \%$ (mean \pm standard deviation, $n$ $=22$ ). Similar results were observed for SFs. Comparison of inhibition curves for sera and SFs with those for aggrecan revealed very good parallelity ensuring accurate epitope measurement (Fig. 1). The KS IgG assay gave very similar results for these analyses (data not shown).

Mouse monoclonal IgM antibody $846(17,18)$ was used in a similar radioimmunoassay to that for $\mathrm{KS}$ using ${ }^{125} \mathrm{I}$-labeled human fetal aggrecan, except that for body fluids a pig anti-rabbit IgM serum was used instead of Protein A-bearing Staphylococcus aureus, which is normally used for tissue samples (17). All assays were performed in triplicate with $50 \mu \mathrm{l}$ of serum per assay tube. The coefficient of variation for interassay variation of the 846 assay of serum was $9.9 \pm 6.5 \%(n=29)$ and intraassay variation was $4.8 \pm 5.1 \%(n=30)$. Spiking experiments of sera involved addition up to $6.25 \mathrm{mg}$ of fetal human aggrecan with good recoveries (mean $109.4 \pm 23.8 \% n=34$ ). Similar results were observed for SF. Due to the low content of the 846 epitope in sera, samples were assayed undiluted. Comparison of inhibition curves for sera and SF with those for aggrecan revealed very good parallelity (Fig. 1). 

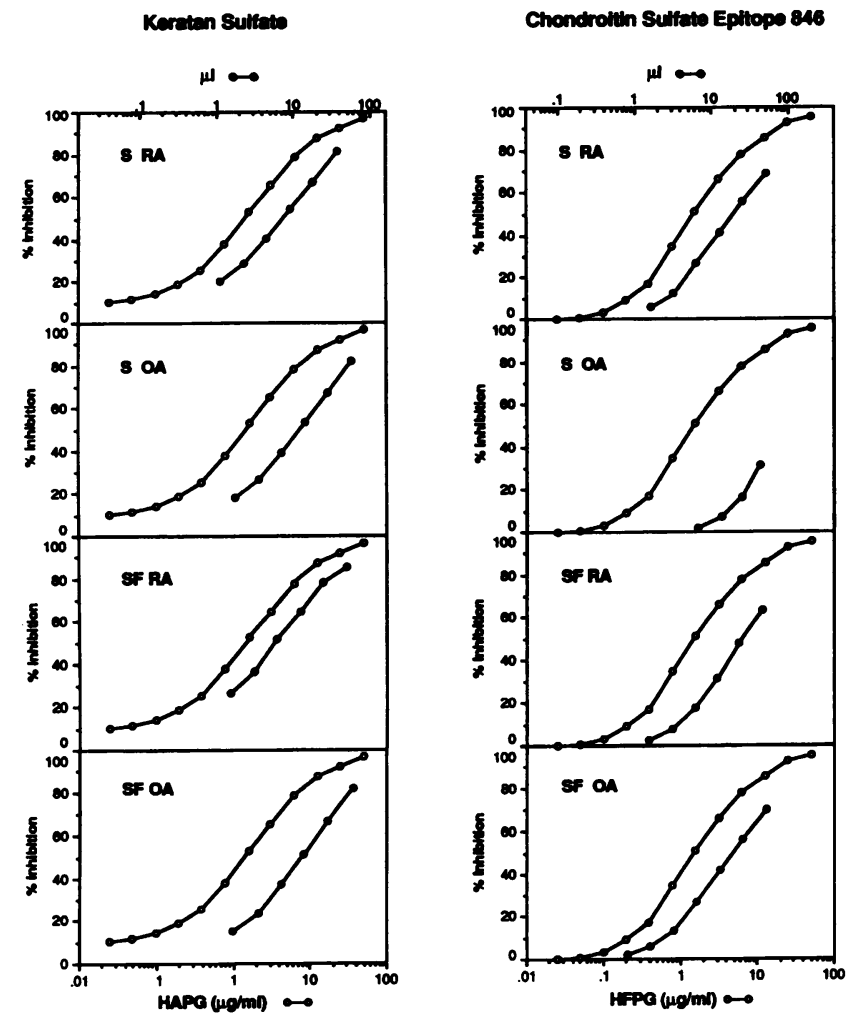

Figure 1. Comparison of inhibition curves for assays of 846 and KS Fab epitopes in sera $(s)$ and synovial fluids $(S F)$ of patients with OA and RA. Human adult (HAPG) and fetal (HFPG) aggrecan standard curves are shown for reference.

Statistical analyses. Means \pm standard deviations were recorded. Mann Whitney analyses of significance were performed when comparing groups. Spearman rank analyses of correlation were used to relate aggrecan markers to each other and to inflammation markers. Correlation coefficients $(r)$, levels of significance $(P)$, and means \pm standard deviations are shown.

\section{Results}

It was not possible to perform all assays on each of the samples obtained, explaining some variation in numbers available for each category of the results.
Concentrations of aggrecan epitopes in sera and SFs

Serum and SF KS. Using the IgG immunoassay to detect primarily the large KS-containing aggrecan core protein fragments, no difference was found between serum levels in the normal control subjects and in those with OA. However, levels in patients with RA were significantly lower than in either of the two groups (Table II). Use of the Fab reagent in the immunoassay, which also detects small KS-bearing fragments, resulted in significantly higher KS serum levels in all groups (especially the control) when compared with the IgG assay of larger fragments $(p<0.001)$, (Table II, Fig. 2). With this Fab assay, we found that serum levels were significantly less in both the $O A$ and RA groups when compared to the normal control subjects. A greater proportion of RA patients (32\%) than OA patients (10\%) had Fab KS levels that were well below the lower limit of normal (Table II). Correlations between the two assays were significant in the serum in all three patient populations, but were higher in RA $(\mathrm{r}=0.74, n=116)$ and $\mathrm{OA}(\mathrm{r}=0.74, n=89)$ than in the normal controls $(r=0.49, n=113)$ where the differences between the two assays were most apparent, due to the presence of smaller KS-containing fragments of core protein in serum. At present there is no indication that these differences could relate to differences in KS chain length.

SF levels of KS were often higher than those in the serum, irrespective of which of the two assays was used, or whether the patients had RA or OA (Table II and III, Fig. 3). In addition, excellent correlations $(p=0.0001)$ between the two assays were observed in both patients with RA $(r=0.94, n=131)$ and OA patients $(r=0.85, n=64)$ (Table II). The ratios of $\mathrm{KS}$ levels in the two body fluids in individual patients were also determined (Table III). These ratios were similar in both diseases, and with both assays, the majority of patients having higher KS levels in the SF than in the serum.

There were no significant correlations between serum and SF levels of KS for each individual assay but serum KS measured by the IgG assay showed a weak correlation with SF KS determined by the Fab assay in OA and the IgG assay in RA (Table IV).

Serum and $S F$ chondroitin sulfate epitope 846. As shown in Table II and Fig. 2, serum levels were slightly higher in OA patients than in normal controls, but much higher in the RA patients. SF levels were very much higher than serum levels in both patient groups, the relative mean ratio between serum and

Table II. Concentrations of Cartilage Proteoglycan Epitopes in Sera and Synovial Fluids

\begin{tabular}{|c|c|c|c|c|}
\hline \multirow[b]{2}{*}{ Body fluid } & \multirow[b]{2}{*}{ Epitope/assay } & \multicolumn{3}{|c|}{ Concentration $\mu \mathrm{g} / \mathrm{ml},(n)$} \\
\hline & & Normal & Osteoarthritis & Rheumatoid arthritis \\
\hline \multirow[t]{4}{*}{ Serum } & KS IgG & $3.16 \pm 1.32(116)$ & $2.84 \pm 1.12(89)$ & $2.47 \pm 1.12(122)$ \\
\hline & KS Fab' & $4.30 \pm 1.13(113)$ & $3.24 \pm 1.56(89)^{9}$ & $3.10 \pm 1.83(116)$ \\
\hline & 846 & $0.09 \pm 0.06(88)$ & $0.11 \pm 0.12(72)^{\S}$ & $0.33 \pm 0.40(50)^{\|}$ \\
\hline & Ratio 846/KS & $0.023 \pm 0.020(88)$ & $0.036 \pm 0.032(50)^{\S}$ & $0.132 \pm 0.137(62)$ \\
\hline \multirow[t]{4}{*}{ Synoval fluid } & KS IgG & - & $6.76 \pm 4.82(71)$ & $5.34 \pm 5.70(135)$ \\
\hline & KS Fab' & - & $5.89 \pm 4.82(64)$ & $5.42 \pm 5.83(131)$ \\
\hline & 846 & - & $1.45 \pm 0.89$ & $1.63 \pm 1.15(91)$ \\
\hline & Ratio 846/KS & - & $0.68 \pm 1.05(38)$ & $1.35 \pm 2.78(76)$ \\
\hline
\end{tabular}

Means \pm standard deviations. Significantly different compared with normal serum or OA synovial fluid. ${ }^{\ddagger} P \leq 0.05,{ }^{\S} P \leq 0.01 ; " \| P \leq 0.001$; ' $P<0.0001$. All the KS Fab values are significantly larger $(P=0.001)$ than the corresponding $\mathrm{KS}$ IgG values for each serum group. Ratios of $846 / \mathrm{KSFab}^{\prime}$ are shown as $\mu \mathrm{g} / \mu \mathrm{g}$. 

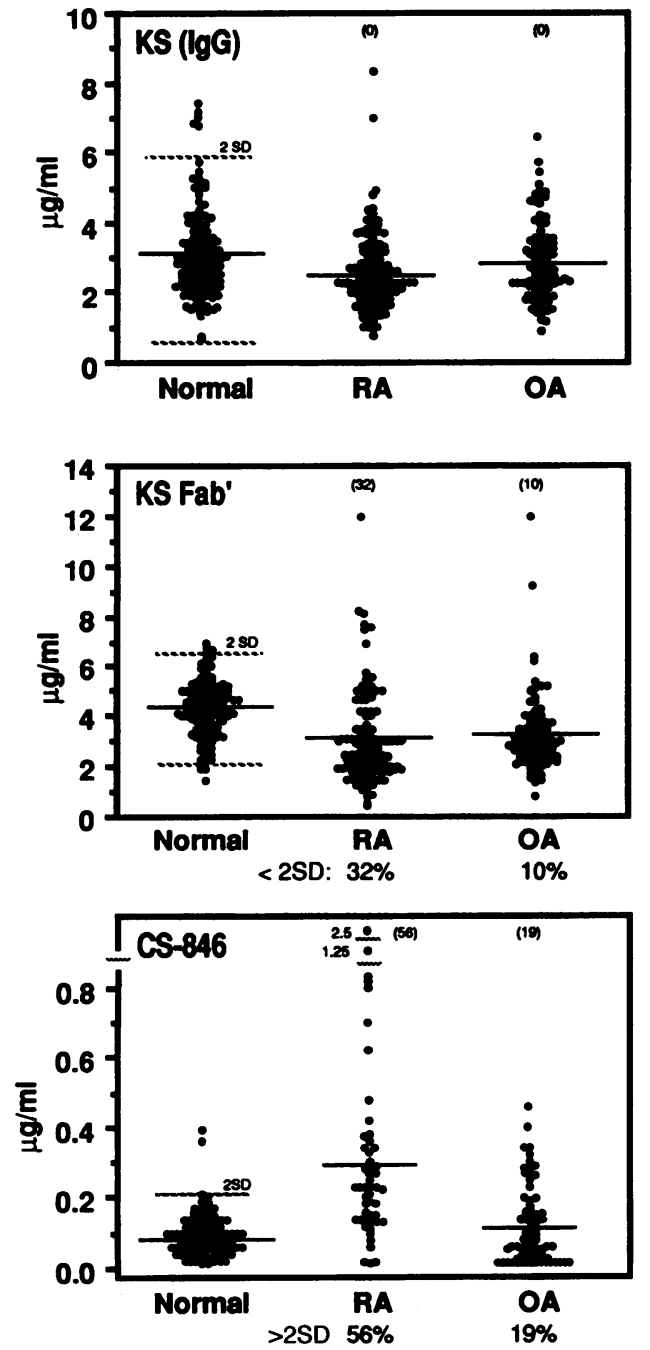

Figure 2. Contents of cartilage aggrecan epitopes in sera of different patients with rheumatoid arthritis $(R A)$, osteoarthritis $(O A)$ and nonarthritic (Normal) persons. The means (solid lines) and twice the standard deviations ( $2 S D$, broken lines) are indicated. The percentage of patients with values greater $(846)$ or less than $(K S)$ twice the SD of the normal group are indicated in parentheses.

SF being greater in OA patients (38) than in RA (8.6) (Table III). Furthermore, every patient in which concurrent serum and SF levels were assayed, showed a higher level of 846 in the SF than in the serum. There was no significant correlation between the serum and SF levels of 846.

\section{Correlations between $K S$ and 846 assays}

Correlations were observed between the KS and 846 epitope levels in both sera and SF (Table IV). A weak but statistically significant positive correlation between serum KS levels, measured by the IgG assay (larger fragments), and serum 846 levels was observed in the sera of patients with OA and in the normal controls but not in RA. Yet the ratio of $846 / \mathrm{KS}$ (Fab assay) epitopes was significantly higher than in the normal ratio in patients with RA (Table II) mainly because of the common increase in serum of 846 epitope and the decrease in KS Fab' in these patients. SF levels of KS, measured by the Fab assay (smaller fragments) and the 846 epitope levels were inversely related in RA and, to a much greater extent, in OA. When the ratio of the 846 epitope to the KS (Fab assay) epitope was examined in SF of these patients, the ratio was higher in RA than OA but this difference was not significant due to wide variations (Table II).

A direct relationship was also observed in RA between serum KS (measured as IgG or Fab assays) and SF 846 epitope.

Correlations between serum aggrecan assays and assays of systemic and joint inflammation

These are illustrated in Table IV, which demonstrates how well one parameter followed another. In patients with RA there was a significant inverse relationship between serum KS (Fab assay) and the acute phase protein orosomucoid. Serum KS (Fab assay) was also inversely correlated with SF MPO (Fig. 4). Moreover, where joint leucocyte count was increased KS levels were reduced (Fig. 5). In contrast, no correlations between KS and acute phase protein were apparent in patients with OA. In RA serum KS (Fab assay) was also indirectly correlated with total joint score. Total joint score also correlated with the ratio of RA serum epitope 846 to KS (Fab assay) epitope in RA showing that the decrease in serum $\mathrm{KS}$ and increase in serum 846 are related to joint disease (Fig. 6). Thus serum KS is reflective of both systemic and local (joint) inflammation.

Correlations between synovial fluid aggrecan assays and measures of local joint inflammation

The data were examined for evidence of correlations between the KS (Fab assay) and 846 epitope levels in the SF and the local measures of inflammation, including clinical evidence of pain, warmth, swelling, and tenderness, the SF total and differential leucocyte counts, and the SF levels of ECP and MPO.

Although individually epitopes 846 and KS (Fab assay) did not correlate with total joint score, the ratio of SF $846 / \mathrm{KS}$ revealed a significant increase, although of relatively low significance, as joint score decreased (Fig. 6). In RA, KS levels increased with elevated leucocyte count (Fig. 5), but aggrecan epitope 846 decreased. The ratio of SF 846 and KS (Fab assay) epitopes was also clearly decreased as leucocyte counts increased (Fig. 7). In contrast, KS levels were reduced in OA when leucocyte count increased or less significantly, when PMN became the dominant cell type (differential count), but there were no changes in 846 epitope in relationship to cell counts or type (differential count).

In RA, there were no observed correlations between either SF KS or 846 and ECP or MPO levels. However, in OA a significant inverse correlation existed for KS (Fab assay) and ECP and MPO concentrations (Table IV) confirming the negative association for KS with the level of intra-articular inflam-

Table III. Ratio of Concentrations of Cartilage Proteoglycan Epitopes in Sera(s) and SF

\begin{tabular}{|c|c|c|c|c|}
\hline \multirow[b]{2}{*}{ Epitope/assay } & \multicolumn{2}{|c|}{ Ratio $\mathrm{SF} / \mathrm{S}(n)$} & \multicolumn{2}{|c|}{$\begin{array}{c}\%>1.0 \\
(\mathrm{SF} / \mathrm{S})\end{array}$} \\
\hline & Osteoarthritis & Rheumatoid arthritis & $\mathrm{OA}$ & RA \\
\hline KS IgG & $2.1 \pm 1.9(49)$ & $2.0 \pm 2.2(94)$ & 61 & 56 \\
\hline KS Fab & $1.7 \pm 1.4(45)$ & $2.3 \pm 2.3(89)$ & 69 & 69 \\
\hline 846 & $38.0 \pm 38.5(30)$ & $8.6 \pm 13.4(37)^{*}$ & 100 & 100 \\
\hline
\end{tabular}

Analyses were made of both synovial fluids and serum in each patient. * Significantly different $(P<0.0001)$ compared with $\mathrm{OA}$ group. 

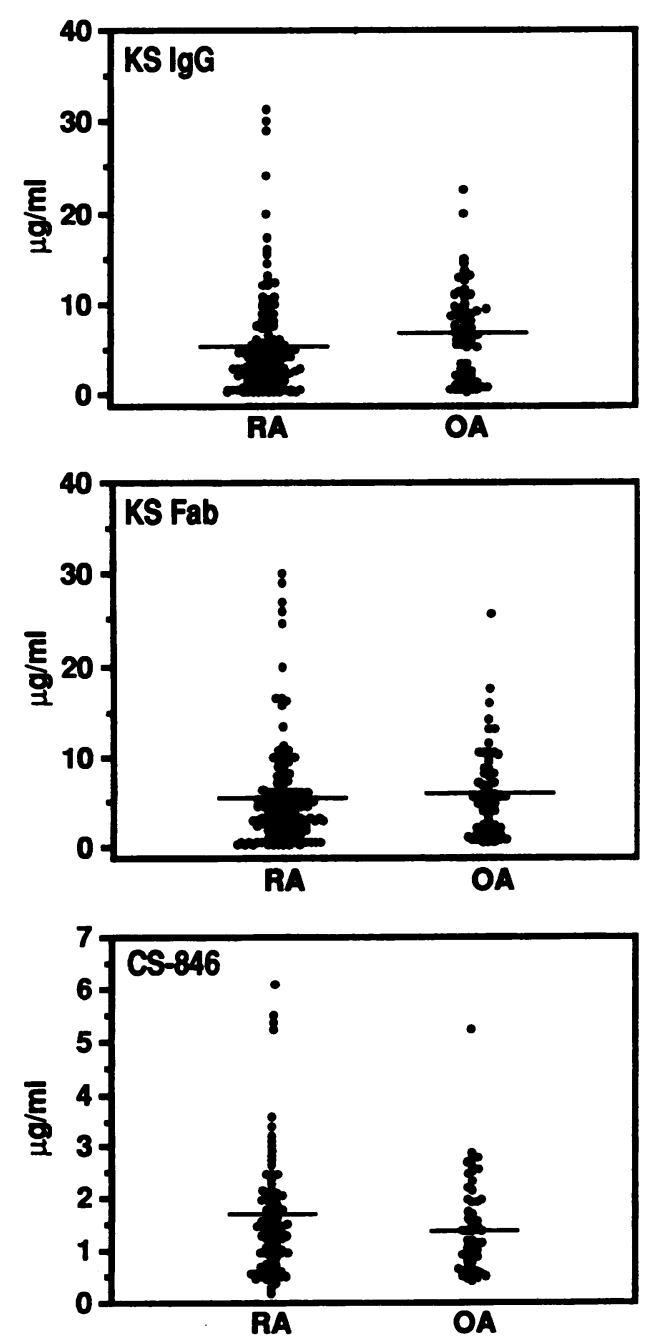

Figure 3. Cartilage aggrecan epitopes in synovial fluids of different patients with rheumatoid arthritis $(R A)$ and osteoarthritis $(O A)$ are shown. Mean values (solid lines) are indicated.

mation (leucocyte and differential counts) in OA. Similar results were obtained with the KS IgG assay (data not shown). No such correlations with the 846 epitope were seen.

\section{Correlations between SF aggrecan epitope levels and evidence of joint destruction}

Correlations were sought between the SF aggrecan levels and the duration and severity of disease in the knee joint from which the fluid had been obtained. Aggrecan levels were therefore related to disease duration, presence or absence of knee instability, and the radiographic features of the index knee joint. No correlations were seen in the RA group. In OA, although numbers were relatively small, there was a statistically significant increase in levels of the 846 epitope in SF with increased disease duration and increased joint space narrowing (cartilage loss) determined from the radiographs (Fig. 6). Moreover, this correlation with disease duration was also, observed when the ratios of 846 and KS (Fab assay) epitopes were examined (Fig. 7).

\section{Discussion}

These studies are motivated by the desire to understand the mechanisms of cartilage degeneration in the two major forms
Table IV. Correlation of Markers in Serum (S) and SF with Each Other and with Clinical Parameters: Spearman Rank Analyses

\begin{tabular}{llccc}
\hline \multicolumn{1}{c}{ Eisease } & \multicolumn{1}{c}{$n$} & KS v KS & p \\
\hline \multicolumn{2}{c}{ KS } & & & \\
\multicolumn{2}{c}{ KS v 846 } & & & \\
OA & S KG IgG v SF KS Fab & 45 & 0.370 & 0.013 \\
RA & S KS IgG v SF KS IgG & 93 & 0.216 & 0.040 \\
& & 70 & 0.290 & 0.018 \\
& & 88 & 0.260 & 0.016 \\
OA & S KS IgG v S 846 & 49 & -0.510 & 0.0004 \\
NA & S KS IgG v S 846 & 90 & -0.230 & 0.030 \\
OA & SF KS Fab v SF 846 & 64 & 0.403 & 0.001 \\
RA & SF KS Fab v SF 846 & 63 & 0.459 & 0.0003 \\
RA & S KS IgG v SF 846 & &
\end{tabular}

KS or $846 \mathrm{v}$ inflammation

$\begin{array}{llccc}\text { RA } & \text { S KS Fab v S orosomucoid } & 110 & -0.217 & 0.024 \\ \text { RA } & \text { S KS Fab v SF MPO } & 43 & -0.366 & 0.018 \\ \text { RA } & \text { S KS Fab v joint score } & 17 & -0.563 & 0.024 \\ \text { OA } & \text { SF KS Fab v SF ECP } & 32 & -0.389 & 0.030 \\ \text { OA } & \text { SF KS Fab v SF MPO } & 34 & -0.492 & 0.005 \\ \text { RA } & \text { S 846/S KS Fab v joint score } & 16 & 0.526 & 0.042 \\ \text { RA } & \text { SF 846/SF KS Fab v joint score } & 26 & 0.444 & 0.027\end{array}$

of arthritis, RA and OA, and to be able to assess changes in cartilage metabolism in vivo. In vitro studies have helped enormously in the understanding of how cytokines, growth factors, and hormones may influence cartilage metabolism, but it is obviously important to develop means of studying and understanding cartilage metabolism in vivo if we are to be able to understand how cartilage metabolism changes in disease. An in vivo approach may also help determine whether therapeutic interventions have beneficial or negative effects on cartilage metabolism. In the present study, we sought to investigate aggrecan metabolism in OA and RA, both systemically (serum) and in diseased joints and to relate the changes to measures of systemic and local joint inflammation and joint damage.

The patients recruited to the study all had definite RA or OA of sufficient severity for them to have been referred to a rheumatology center, and of sufficient activity to require aspiration of SF from a knee joint. It is therefore not surprising that there were relatively few patients with recent onset disease, and that there was active inflammation in all those with RA; factors that put some restrictions on our ability to relate changes to disease duration and severity, or the extent of the inflammatory response in RA. All our patients were on some form of drug therapy, including a wide range of analgesic, anti-inflammatory, and anti-rheumatic drugs, and it was not possible to control for the possible effects of these agents on cartilage metabolism. However, because of the known inhibitory effects of steroids on chondrocytes metabolism $(35,36)$, patients who had received a local steroid injection to the index knee within the last three months were excluded.

It has been reported that serum levels of aggrecan fragments bearing KS, measured by ELISA immunoassay of a highly sulfated domain of KS by the antibody 5D4, are elevated in patients with OA (22-25). However, more recent data has not con- 

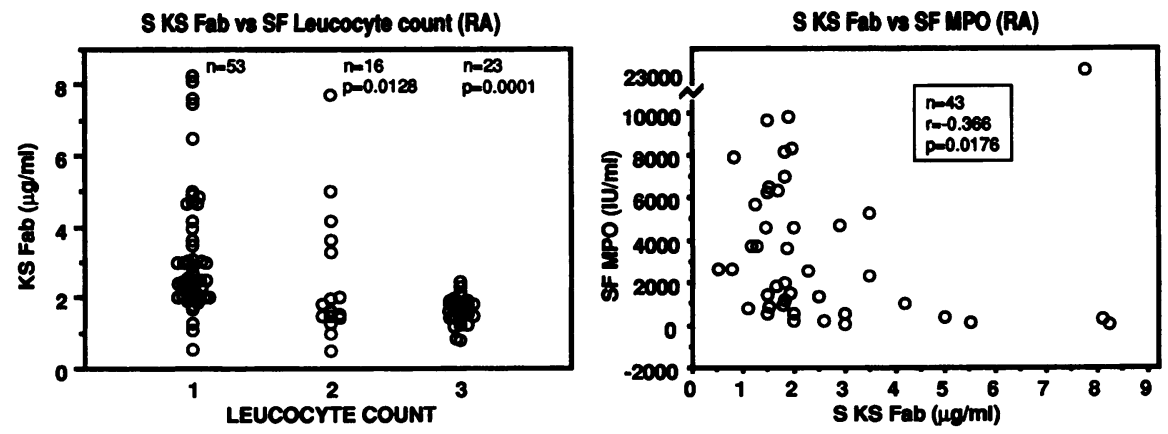

Figure 4. Serum $(S)$ KS levels (determined by KS Fab' assay) are reduced when leucocyte count or myeloperoxidase contents increase in RA.

firmed an increase of KS levels in OA (30). Using our antibody to $\mathrm{KS}$, which exhibits very similar reactivity to antibody 5D4 (31) in assays that detect either primarily large fragments (IgG assay), or both large and small fragments (Fab assay), we could find no evidence of serum elevations in patients with $\mathrm{OA}$ in comparison with a large control group. There was a reduction in serum $\mathrm{KS}$ in patients compared with controls in the RA group. This was most apparent when the KS Fab assay (which detects both large and the small fragments) was used. The reduction in serum KS levels in RA was shown to be due to a reduction in the absolute content rather than the size of fragments present in arthritic samples compared with normals; the normal sera would appear to contain more of the smaller fragments which are not detected by the assay utilizing intact IgG. Whether these differences could be due to changes in KS chain length is unclear at present. Inflammation may be the cause of the reduction in serum content of the KS epitope. Our earlier work (29) and a more recent investigation (30) both revealed an inverse correlation between serum $\mathrm{KS}$ and acute phase proteins in RA, a finding that has been confirmed for OM in this investigation. Serum TNFa levels are also indirectly related to $\mathrm{KS}$ levels in RA (32). Moreover, in RA we also noted that as leukocyte joint fluid count or MPO or joint score increased so serum levels decreased. Therefore increased inflammation both systemically and in joints negatively influences serum KS levels.

KS levels in the SF were also related to the degree of local inflammation, as measured by cell counts and the release of ECP and MPO. As expected, most OA joints had little evidence of synovitis, but in those which did have an elevation of leucocyte counts, or in which PMN content or MPO or ECP levels were increased, there was a reduced level of KS fragments. Thus in OA, the local joint relationships resembled those observed between serum KS levels and markers of systemic and local joint inflammation in RA. The situation was quite different in RA SF, in which there was a direct relationship between KS levels and leucocyte counts, as seen for aggrecan in other studies of inflammatory arthritis (37), but no relationship between KS levels and ECP/MPO. However, whereas OA joints could be clearly differentiated into those with and without significant synovitis, all RA joints exhibited severe active inflammation, making it difficult to differentiate the effect of varying synovitis on local cartilage metabolism in this disease.

The observations of an inverse relationship between inflammation and KS in OA may result from an inhibitory effect of inflammation on cartilage aggrecan synthesis. The amount of KS released by degradation will be substrate dependant. But if inflammation were to inhibit synthesis of a pool of KS-rich
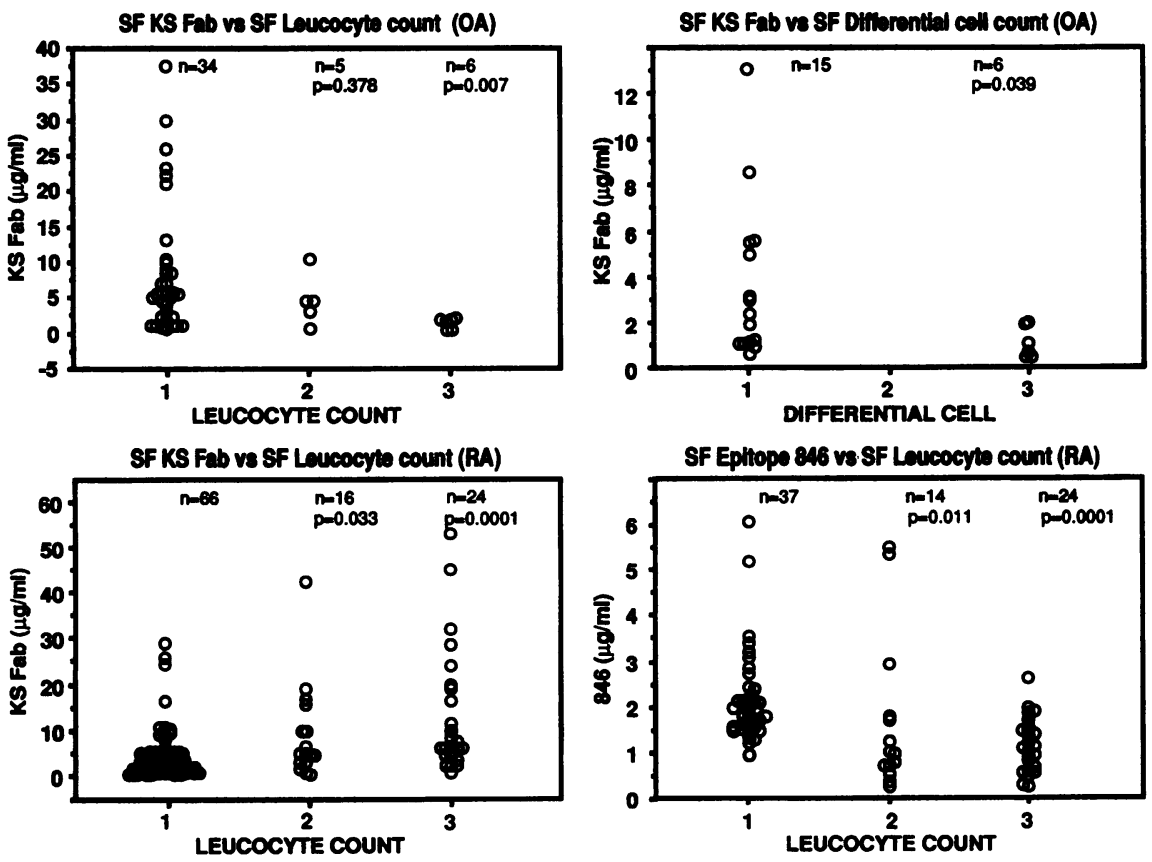

Figure 5. Relationship of synovial fluid ( $S F)$ aggrecan epitopes to joint inflammation in $O A$ and RA measured as leucocyte count $(O A$ and $R A)$ and differential count $(O A)$. KS levels were determined by KS Fab assay. Significance ( $P$ value) are shown for $n$ samples. 

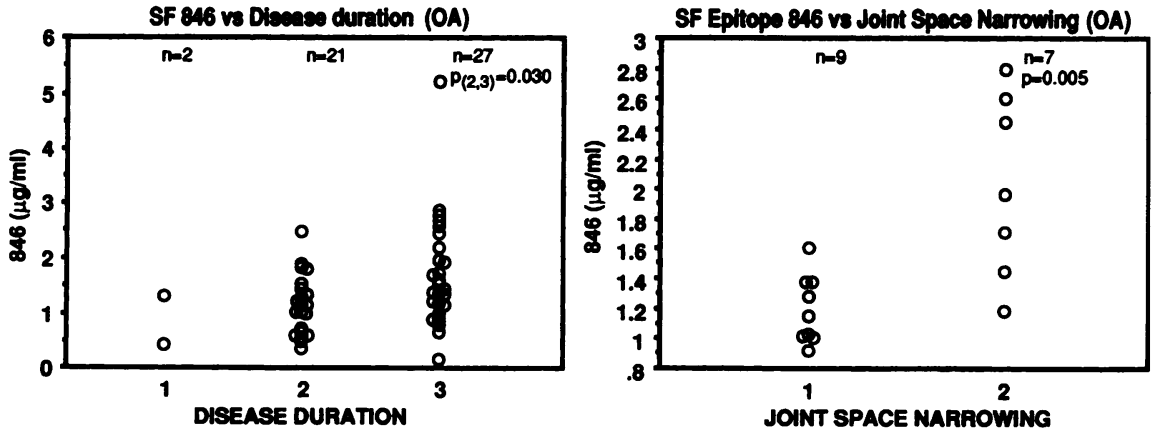

Figure 6. Relationship of synovial fluid 846 epitope to disease duration and joint space narrowing in OA.

proteoglycan that was susceptible to degradation shortly after synthesis, then there would be less aggrecan to be degraded, and less KS released. It is well known that inflammatory mediators such as IL-1 and TNFa can potently inhibit synthesis in vitro, and in vivo, at levels far less than those required to induce degradation (10-14). Previous investigations have indicated that IL-lb levels in OA SF are much lower than those found in RA whereas those of TNFa are similar (38). Others have noted increased levels of TNF-a in SF of those with active RA compared to fluids from OA patients (39). It is therefore possible that the mild synovitis in OA results in release of cytokines in a concentration that can inhibit local proteoglycan synthesis, whereas the much more severe inflammation of RA results in a constant high level of cytokines, resulting in both inhibition of synthesis and stimulation of degradation of cartilage matrix molecules and hence the increase in local KS levels. Thus systemically in RA and locally in joints in OA the release of KS may be a consequence of degradation of newly/recently synthesized proteoglycan influenced by lower levels of cytokines that inhibit synthesis, of substrate leading to reduced degradation products. The indirect correlation of serum TNFa levels with KS in RA (32) supports this conclusion. This contrasts to joint inflammation in RA where we are likely observing in- creased degradation of "resident" aggrecan molecules when we measure increased levels of KS.

In apparent contradiction of this hypothesis for RA is the inverse correlation between proteoglycan and IL-1 levels that has been observed in SF of patients with RA (40). This is the type of result predicted for OA SF. Perhaps these patients had less active inflammation and SF IL-1 levels were lower and inhibited synthesis rather than influencing degradation. The observation that in normals and OA serum KS levels directly correlate with serum 846 levels (which may be a measure of synthesis of aggrecan [see below]) would also indicate that serum $\mathrm{KS}$ is indirectly reflective of proteoglycan synthesis and includes molecules synthesized in diseased joints in RA, since serum KS in RA was correlated with synovial fluid 846. More work on RA patients with relatively quiescent disease and a search for relationships with cytokines and enzyme levels in SF and sera would help to investigate this hypothesis.

The elevation in synovial KS over serum levels in the majority of patients with both OA and RA clearly indicates that SF content primarily reflects local cartilage metabolism. This elevation was even more striking for the CS epitope 846 , where all patients, irrespective of disease, exhibited SF levels which were much greater than serum levels: the ratios between SF
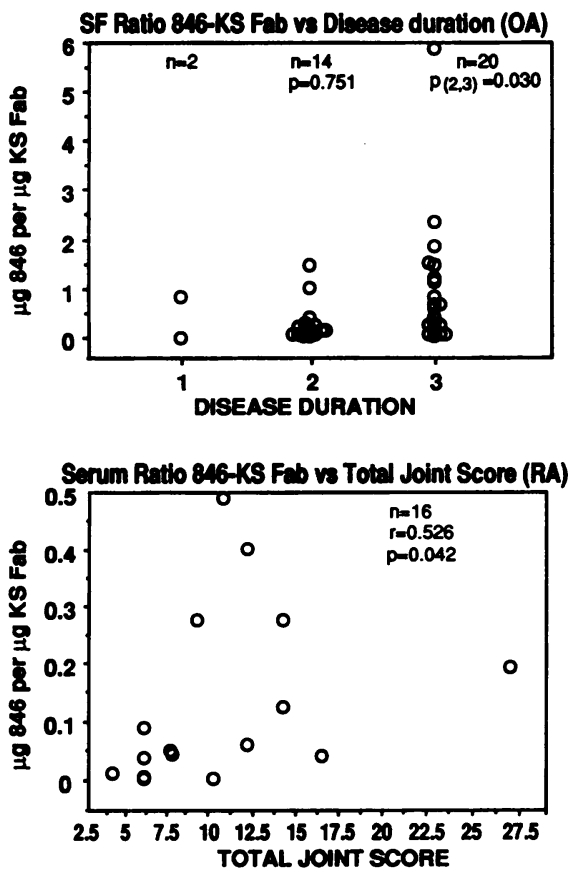
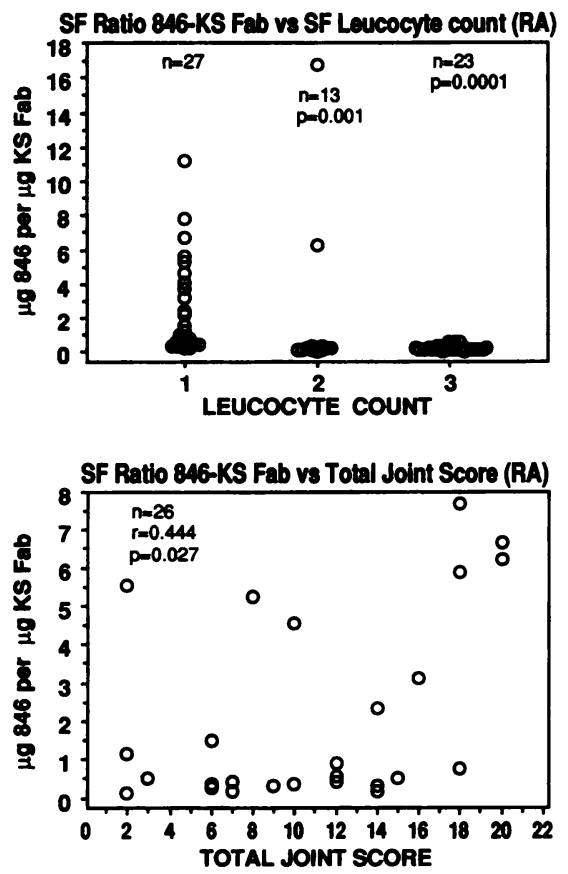

Figure 7. Relationships of ratio of $846 / \mathrm{KS}$ Fab epitopes $(\mu \mathrm{g} / \mu \mathrm{g})$ in serum and SF to inflammation and clinical parameters. 
and serum levels were much higher than with the KS epitope. Thus SF levels of the 846 epitope more clearly reflect local abnormalities in cartilage metabolism.

Recent studies have suggested that the CS epitope 846 is likely to be a marker of aggrecan synthesis rather than degradation (17), although this still requires direct confirmation. The epitope is present on the largest aggrecan molecules: in normal cartilages on those molecules which exhibit complete aggregation with hyaluronic acid. Ordinarily only about $50-55 \%$ of the molecules aggregate. This is likely reduced due to damage to the region of the molecule (the G1 globular domain) that binds to hyaluronic acid (17). Levels of the 846 epitope in OA articular cartilages are elevated over those in normals (17). Thus, its striking elevation in SF of patients with OA and to a lesser degree with RA coupled with increased disease duration and joint space narrowing likely reflects increased metabolic changes. These are probably at the level of aggrecan synthesis which is increased in OA $(1,2)$ and which in RA joints may be inhibited at the level of synthesis when leucocyte counts increase, in view of the indirect correlation with the 846 epitope. Circulating levels of the 846 epitope, both absolute levels and these related to $\mathrm{KS}$, were commonly elevated in patients with RA and to a lesser extent in OA. This may relate to the release of this epitope from cartilages in diseased joints since the $846 /$ $\mathrm{KS}$ ratio correlated with total joint score in RA. OA, unlike RA, usually affects likely a smaller number of joints, and thus a smaller proportion of total body cartilage would likely contribute to 846 epitope levels in serum.

In contrast, SF levels of 846 were inversely related to KS Fab epitope levels in both disorders. This would be expected if local KS production results from aggrecan degradation, and 846 from synthetic activity in contrast to serum where KS and 846 are directly correlated in normals and in OA. The inverse relationship of the SF 846 epitope to cell and differential counts in RA fluids would also argue in favor of 846 being a marker of synthesis, since inflammatory cytokines can result in reduced synthesis in vivo (10). The decrease in the $846 / \mathrm{KS}$ ratio in RA SF with increased leucocyte count points to a possible increase of degradative over synthetic activity whereas in OA joints, the increase in this ratio with disease duration in OA reflects a shift towards synthesis in more advanced disease as we observed before (17).

The elevation of 846 epitope in sera of patients with RA and its correlation with joint involvement in RA (including the $846 / \mathrm{KS}$ ratio) indicate that its measurement in serum may be of real value in studying the effect of disease on joint cartilage metabolism in RA. In fact in more recent studies we have observed a striking reduction in serum levels of this epitope in patients with early RA which exhibit rapid erosive disease compared with those with slow erosive disease (Saxne, T., D. Heinegård, M. Ionescu, and A. R. Poole, manuscript in preparation ). Again this may reflect impaired synthesis of aggrecan.

In conclusion, these studies raise important questions concerning alterations in cartilage aggrecan metabolism in arthritis, how it may be differently affected by inflammation in RA and $\mathrm{OA}$ and how it may be measured in future. They demonstrate the potential for using measurement of aggrecan epitopes in body fluids as indicators of disease activity and in particular identify serum 846 epitope as a particularly valuable index of disease activity in RA.

\section{Acknowledgments}

We thank A. Wheeler for help in processing the manuscript and Mark Lepik and Jane Wishart for the artwork.
This study was funded by the Arthritis and Rheumatism Council (P. A. Dieppe), the Shriners of North America, and Pharmacia Diagnostics (A. R. Poole).

\section{References}

1. Mankin H. J., and L. Lippiello. 1971. The glycosaminoglycans of normal and arthritic cartilage. J. Clin. Invest. 50:1712-1719.

2. Thompson, R., and T. Oegema. 1979. Metabolic activity of articular cartilage in osteoarthritis. J. Bone Jt. Surg. 61 A:407-416.

3. Sandy, J., M. Adams, M. Billingham, A. Plaas, H. Muir. 1984. In vivo and in vitro stimulation of chondrocyte biosynthetic activity in early experimental osteoarthritis. Arthritis Rheum. 27:388-397.

4. Schwartz, E., C. Leveille, J. Stevens, and W. Oh. 1981. Proteoglycan structure and metabolism in normal and osteoarthritic cartilage of guinea pigs. Arthritis Rheum. 24:1528-1529.

5. Eastgate, J. A., N. C. Wood, F. S. Di Giovine, J. A. Symons, F. M. Grinlinton, and G. W. Duff. 1988. Correlation of plasma interleukin 1 levels with disease activity in rheumatoid arthritis. Lancet. 2:706-709.

6. Hopkins, S. J., M. Humphreys, and M. I. V. Jayson. 1988. Cytokines in synovial fluid I. The presence of biologically active and immunoreactive IL-1. Clin. Exp. Immunol. 72:422-427.

7. Saklatvala, J., L. M. C. Pilsworth, S. J. Sarsfield, J. Gavrilovic, and J. K. Heath. 1984. Pig catabolin is a form of interleukin 1. Cartilage and bone resorb, fibroblasts make prostaglandin and collagenase, and thymocyte proliferation is augmented in response to one protein. Biochem. J. 224:461-466.

8. Pettipher, E. R., G. A. Higgs, and B. Henderson. 1986. Interleukin 1 induces leukocyte infiltration and cartilage proteoglycan degradation in the synovial joint. Proc. Natl. Acad. Sci. USA. 83:8749-8753.

9. Poole, A. R. Cartilage in health and disease. In Arthritis and Allied Conditions. A Textbook of Rheumatology, 12th ed. D. McCarthy and W. Koopman, editors. Lea \& Febiger, Malvern, PA. 279-333.

10. van der Loo, A. A. J., and W. B. van den Berg. 1990. Effects of murine recombinant interleukin 1 on synovial joints in mice: measurement of patellar cartilage metabolism and joint inflammation. Ann. Rheum. Dis. 49:238-245.

11. Arner, E. C., and M. A. Pratta. 1989. Independent effects of interleukin1 on proteoglycan breakdown, proteoglycan synthesis, and prostaglandin $\mathrm{E}_{2}$ release from cartilage in organ culture. Arthritis Rheum. 32:288-297.

12. Goldring, M. B., H. Birkhead, L. J. Sandell, T. Kimura, and S. M. Krane 1988. Interleukin 1 suppresses expression of cartilage-specific types II and IX collagens and increases types I and III collagens in human chondrocytes. J. Clin. Invest. 82:2026-2037.

13. Tyler, J. A. 1985. Articular cartilage cultured with catabolin (pig interleukin 1) synthesizes a decreased number of normal proteoglycan molecules. Biochem. J. 227:869-878.

14. Tyler, J. A., and H. P. Benton. 1988. Synthesis of type II collagen is decreased in cartilage cultured with interleukin 1 while the rate of intracellular degradation remains unchanged. Collagen Relat. Res. 8:393-405.

15. Roughley, P. J., and J. S. Mort. 1986. Aging and the aggregating proteoglycans of human articular cartilage. Clinical Sci. (Lond.). 71:337-344.

16. Webber, C., T. T. Glant, P. J. Roughley, and A. R. Poole. 1987. The identification and characterization of two populations of aggregating proteoglycans of high buoyant density isolated from post-natal human articular cartilages of different ages. Biochem. J. 248:735-740.

17. Rizkalla, G., A. Reiner, E. Bogoch, and A. R. Poole. 1992. Studies of the articular cartilage proteoglycan aggrecan in health and osteoarthritis: evidence for molecular heterogeneity and extensive molecular changes in disease. J. Clin. Invest. 90:2268-2277.

18. Glant, T. T., K. Mikecz, P. J. Roughley, E. Buzas, and A. R. Poole 1986. Age-related changes in protein-related epitopes of human articular-cartilage proteoglycans. Biochem. J. 236:71-75.

19. Caterson, B., F. Mahmoodian, J. M. Sorrell, T. E. Hardingham, M. T. Bayliss, S. L. Carney, A. Ratcliffe, and H. Muir. 1990. Modulation of native chondroitin sulphate structure in tissue development and in disease. J. Cell. Science. 97:411-417.

20. Visco, D. M., B. Johnstone, M. A. Hill, G. A. Jolly, and B. Caterson. 1994. Immunohistochemical analysis of 3-B-3 (-) and 7-D-4 epitope expression in canine OA. Arthritis Rheum. In press.

21. Witter, J., P. J. Roughley, C. Webber, N. Roberts, E. Keystone, and A. R. Poole. 1987. The immunologic detection and characterization of cartilage proteoglycan degradation products in synovial fluids of patients with arthritis. Arthritis Rheum. 30:519-529.

22. Thonar, E. J.-M. A., M. E. Lenz, G. K. Klintworth, B. Caterson, L. M. Pachman, P. Glickman, R. Katz, and K. E. Kuettner. 1985. Quantification of keratan sulfate in blood as a marker of cartilage catabolism. Arthritis Rheum. 28:1367-1376

23. Sweet, M. B. E., A. Coelho, C. M. Schnitzler, T. J. Schnitzer, M. E. Lenz, I. Jakim, K. E. Kuettner, and E. J-M. A. Thonar. 1988. Serum keratan sulfate levels in osteoarthritis patients. Arthritis Rheum. 31:648-652.

24. Mehraban, F., C. K. Finegan, and R. W. Moskowitz. 1991. Serum keratan 
sulfate. Quantitative and qualitative comparisons in inflammatory versus noninflammatory arthritides. Arthritis Rheum. 34:383-392.

25. Campion, G. V., F. McCrae, T. J. Schnitzer, M. E. Lenz, P. A. Dieppe and E. J-M. A. Thonar. 1991. Levels of keratan sulfate in the serum and synovial fluid of patients with osteoarthritis of the knee. Arthritis Rheum. 34:1254-1259.

26. Saxne, T., D. Heinegård, and F. A. Wollheim. 1987. Cartilage proteoglycans in synovial fluid and serum in patients with inflammatory joint disease. Relation to systemic treatment. Arthritis Rheum. 30:972-979.

27. Ratcliffe, A., M. Doherty, R. N. Maini, and T. E. Hardingham. 1988. Increased concentrations of proteoglycan components in the synovial fluids of patients with acute but not chronic joint disease. Ann. Rheum. Dis. 47:826-832.

28. Dahlberg, L., L. Ryd, D. Heinegård, and L. S. Lohmander. 1992. Proteoglycan fragments in joint fluid. Influence of arthritis and inflammation. Acta. Orthop. Scand. 63:417-423.

29. Poole, A. R., J. Witter, N. Roberts, F. Piccolo, R. Brandt, J. Paquin, and M. Baron. 1990. Inflammation and cartilage metabolism in rheumatoid arthritis. Studies of the blood markers hyaluronic acid, orosomucoid, and keratan sulfate. Arthritis Rheum. 33:790-799.

30. Spector, T. D., L. Woodward, G. M. Hall, A. Hammond, A. Williams, M. G. Butler, I. T. James, D. J. Hart, P. W. Thompson, and D. L. Scott. 1992 Keratan sulphate in rheumatoid arthritis, and inflammatory diseases. Ann. Rheum. Dis. 51:1134-1137.

31. Poole, A. R., C. Webber, A. Reiner, and P. J. Roughley. 1989. Studies of a monoclonal antibody to skeletal keratan sulphate. Importance of antibody valency. Biochem. J. 260:849-856.

32. Manincourt, D-H., R. Triki, K. Fukuda, J-P. Devogelaer, C. Nagant de Deuxchaisnes, and E. J-M. A. Thonar. 1993. Levels of circulating tumor necrosis factor a and interleukin-6 in patients with rheumatoid arthritis. Relation- ship to serum levels of hyaluronan and antigenic keratan sulfate. Arthritis Rheum. 36:490-499.

33. Arnett, F. C., S. M. Edworthy, D. A. Block, D. J. McShane, J. F. Fries, N. S. Cooper, L. A. Healey, S. R. Kaplan, M. H. Liang, H. S. Luthra, T. A. Medsger Jr., D. M. Mitchell, D. H. Neustadt, R. S. Pinals, J. G. Schaller, J. T. Sharp, R. L. Wilder, and G. G. Hunder. 1988. The American Rheumatism Association 1987 revised criteria for the classification of rheumatoid arthritis. Arthritis Rheum. 31:315-324.

34. Thompson, P. W., A. J. Silman, J. R. Kirwan, and H. L. Currey. 1987. Articular indices of joint inflammation in rheumatoid arthritis-correlation with the acute phase response. Arthritis Rheum. 30:618-623.

35. Mankin, H. J., and K. A. Conger. 1966. The acute effects of intra-articular hydrocortisone on articular cartilage in rabbits. J. Bone Jt. Surg. 48A:1383-1388.

36. Chandler, G. N., and V. Wright. 1958. Deleterious effect of intra-articular hydrocortisone. Lancet. ii:661-663.

37. Saxne, T., D. Heinegård, and F. A. Wollheim. 1986. Therapeutic effects on cartilage metabolism in arthritis as measured by release of proteoglycan structures into the synovial fluid. Ann. Rheum. Dis. 45:491-497.

38. Westacott C. I., J. T. Whicher, I. C. Barnes, D. Thompson, A. J. Swan, and P. A. Dieppe. 1990. Synovial fluid concentrations of five different cytokines in theumatic diseases. Ann. Rheum. Dis. 49:676-681.

39. Yoshida, K., K. Kobayashi, N. Yamagata, H. Iwabuchi, T. Katsura, S. Sugihara, M. Negishi, H. Ide, Y. Mori, and T. Takahashi. 1992. Inflammatory cytokines and enzymes in synovial fluid of patients with rheumatoid arthritis and other arthritides. Int. Arch. Allergy Immun. 98:286-292.

40. Saxne T., S. Di Giovine, D. Heinegånd, G. W. Duff, and F. A. Wollheim 1988. Synovial fluid concentrations of interleukin-1b and proteoglycans are inversely related. J. Autoimmunity. 1:373-380. 